\title{
Yet a Lot to Consider Regarding Cognition in Multiple Sclerosis
}

\author{
Nathalie Ehrle ${ }^{1-3^{*}}$ and Serge Bakchine ${ }^{1-3}$ \\ ${ }^{1}$ Hôpital Maison-Blanche, Service de Neurologie, France \\ ${ }^{2}$ Equipe de Neuropsychologie et Cognition auditive, Laboratoire de Neurosciences Fonctionnelles et Pathologies EA 4559, Université de Lille-Nord de France, France \\ ${ }^{3}$ CMRR de Champagne-Ardenne, Reims, France
}

*Corresponding author: Nathalie Ehrlé, Hôpital Maison-Blanche (Service de neurologie) 45, rue Cognacq-Jay 51092 Reims Cedex, France; Tel: (00 33 ) 326783964 ; Fax: (00 33) 326784319; E-mail: nehrle@chu-reims.fr

Received date: May 31, 2014, Accepted date: June 2, 2014, Published date: June 6, 2014

Copyright: (c) 2014 Ehrlé N, et al. This is an open-access article distributed under the terms of the Creative Commons Attribution License, which permits unrestricted use, distribution, and reproduction in any medium, provided the original author and source are credited.

\section{Editorial}

Cognitive impairments were reported in descriptions of Multiple Sclerosis (MS) as early as Jean-Martin CHARCOT's first descriptions by 1868 . However, there were only very few systematic studies in this domain until the contribution from Stephen M. Rao, twenty years ago. Since the introduction of specific standardized tools, such as the BRB [1], clinicians have become aware of neuropsychological consequences of this disease and consequently, have been able to embark on prospective and comparative studies that tried to characterize cognitive deficits in the various forms and stages of MS.

Progress so far, however, is modest and much remains to be done. Still lacking are robust markers allowing prediction of poor intellectual outcome, and indeed the evolution of cognitive impairment and motor or sensory disability appear to be fairly independent, except for the link regularly found between cognitive slowing and EDSS [2]. An important issue is the frequent underestimation or minimization of the heterogeneity of neuropsychological impairment in MS patients. This is related to the strong temptation to apply to MS studies approaches which were previously successful in neurodegenerative brain diseases in elderly patients (such as Alzheimer's and related disorders). In these populations, clinicians were able to identify relatively stable patterns of cognitive and/or behavioural dysfunction related to the regular involvement of certain brain areas (such as "hippocampic memory profile" and apathy for Alzheimer's disease, prominent dysexecutive impairment associated with disorders in social cognition in frontotemporal dementia). In a given patient, the demonstration of such a pattern may be very suggestive of a specific disease even at an early stage. In MS such a link is missing, as the number and location of plaques may vary considerably from one patient to another, without regular clusters of sites. Moreover, while the mapping of cognitive functions to cortical areas is well underway, less is known about the cognitive functions of subcortical white matter pathways. Taken together, one may question the ability of the short cognitive batteries widely used in MS studies to capture more subtle patterns of performances which may be hidden behind mean tendencies. In the recent years, an effort has been made to incorporate more demanding tests in the MS batteries. It seems also mandatory to incorporate scoring methods that aim to identify neuropsychological patterns specific of MS. In this issue of the Journal, we report one such effort, which specifically was able to highlight a link between cognitive slowing and memory impairment in MS [3]. Our results suggest the importance of further systematic characterization of cognitive impairment in MS by developing and using tests targeting specific cognitive processes. Results can then be systematically confronted with brain imaging data in order to identify clusters of correlations between damaged sites and cognitive impairment type and severity. White matter sites with high impact on cognitive worsening may be then more easily identified.

Yet another promising trail, that deserves to be followed, is the recent demonstration of significant impairment of social cognition abilities (emotion processing, theory of mind...) in MS patients, even at early stages [4]. Such results indicate that assessment of social cognition should be systematically assessed in addition to traditional evaluation of higher brain functions. An essential step for the future will be to study relative contribution of social cognition abilities and "cold" cognition to social performances in daily life. Similar studies may help to identify patients at risk to develop behavioural or psychological disorders.

\section{References}

1. Rao SM, Leo GJ, Bernardin L, Uverzagt F (1991) Cognitive dysfunction in multiple sclerosis I: frequency of patterns and predictions. Neurology 41: 685-691.

2. Brochet B, Deloire MS, Bonnet M, Salort-Campana E, Ouallet JC et al. (2008) Should SDMT substitute for PASAT in MSFC? A 5-year longitudinal study. Mult Scler 14:1242-9.

3. Amaya S, Serge B, Igor S, Diana O, Nathalie E (2014) Abnormal Long-Term Episodic Memory Profiles in Multiple Sclerosis?. J Mult Scler 1: 105.

4. Jehna M, Langkammer C, Wallner-Blazek M, Neuper C, Loitfelder M, et al. (2011) Cognitively preserved MS patients demonstrate functional differences in processing neutral and emotional faces. Brain Imaging Behav. 5: 241-251. 